\title{
Gate energy efficiency and negative capacitance in ferroelectric 2D/2D TFET from cryogenic to high temperatures
}

\author{
Sadegh Kamaei $\mathbb{D i D}^{1 凶}$, Ali Saeidi ${ }^{1}{ }^{1}$, Carlotta Gastaldi ${ }^{1}$, Teodor Rosca ${ }^{1}$, Luca Capua ${ }^{1}$, Matteo Cavalieri ${ }^{1}$ and Adrian M. lonescu $\mathbb{D}^{1}$
}

We report the fabrication process and performance characterization of a fully integrated ferroelectric gate $s t a c k$ in a WSe $/ \mathrm{SnSe}_{2}$ Tunnel FETs (TFETs). The energy behavior of the gate stack during charging and discharging, together with the energy loss of a switching cycle and gate energy efficiency factor are experimentally extracted over a broad range of temperatures, from cryogenic temperature $(77 \mathrm{~K})$ up to $100{ }^{\circ} \mathrm{C}$. The obtained results confirm that the linear polarizability is maintained over all the investigated range of temperature, being inversely proportional to the temperature $T$ of the ferroelectric stack. We show that a lower-hysteresis behavior is a sine-qua-non condition for an improved energy efficiency, suggesting the high interest in a true NC operation regime. A pulsed measurement technique shows the possibility to achieve a hysteresis-free negative capacitance (NC) effect on ferroelectric 2D/2D TFETs. This enables sub- $15 \mathrm{mV} \mathrm{dec}^{-1}$ point subthreshold slope, $20 \mathrm{mV} \mathrm{dec}^{-1}$ average swing over two decades of current, $\mathrm{l}_{\mathrm{ON}}$

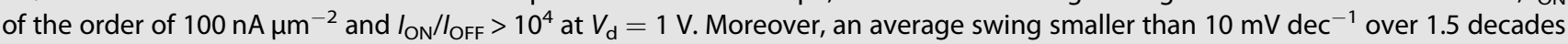
of current is also obtained in a NC TFET with a hysteresis of $1 \mathrm{~V}$. An analog current efficiency factor, up to 50 and $100 \mathrm{~V}^{-1}$, is achieved in hysteresis-free NC-TFETs. The reported results highlight that operating a ferroelectric gate stack steep slope switch in the NC may allow combined switching energy efficiency and low energy loss, in the hysteresis-free regime.

npj 2D Materials and Applications (2021)5:76; https://doi.org/10.1038/s41699-021-00257-6

\section{INTRODUCTION}

The scaling of complementary metal-oxide-semiconductor (CMOS) is facing several fundamental and technical challenges due to the inability to remove the heat generated in the switching process ${ }^{1}$. This results in jeopardizing the performance of aggressively scaled CMOS technology nodes $s^{2,3}$. The origin of this issue can be traced back to the inability to scale the power supply, which is limited by the physic of thermionic injection of carriers. Thus, the subthreshold slope (SS) can not be any lower than $60 \mathrm{mV}$ per decade at room temperature (Boltzmann limit). This impedes a fundamental limit to lowering the dynamic power consumption of a transistor. One of the possible solutions to overcome the Boltzmann limit is to rely on other mechanisms of carrier injection to break through the barrier ${ }^{4-6}$. Tunneling field effect transistors (TFETs) have been proposed to overcome this thermionic limit of SS by exploiting the Band-to-Band tunneling (BTBT) of carriers as the charge transportation mechanism ${ }^{4}$. The use of conventional three-dimensional semiconductors (like silicon, germanium and III-V materials) as TFETs have shown sub-60 mV per decade subthreshold swing at room temperature ${ }^{4,7}$. However, the presence of band-tail states and trap assisted tunneling paths in such devices would drastically deteriorate the turn-on slope ${ }^{8,9}$

Recently, two-dimensional (2D) materials come to the forefront with their excellent electronic properties and the possibility of assembling atomically sharp van der Waals heterojunctions ${ }^{10}$, making them promising candidates for replacement of conventional TFETs and silicon. Although there have been several reports of tunneling in 2D-based heterojunctions with negative differential resistance (NDR) observed at room temperature or cryogenic ${ }^{11-15}$, this type of devices suffer from poor SS. Among the reported configuration of 2D/2D TFETs ${ }^{11,13-19}, \mathrm{WSe}_{2} / \mathrm{SnSe}_{2}$ heterostructure has recently attracted a great deal of attention due to its near broken band alignment which enables BTBT. The band alignment of the $\mathrm{WSe}_{2} / \mathrm{SnSe}_{2}$ heterostructure has been derived by density functional theory (DFT) calculations that shows a type III, broken gap with an expected energy overlap of 26 $\mathrm{meV}^{20}$. Moreover, the peculiar electronic properties of the two members of transition metal dichalcogenide (TMD) constituting this configuration make this system more favorable. Tunability of $\mathrm{WSe}_{2}$ for both electron and hole transport ${ }^{21,22}$ with relatively high mobility $^{23}$, presents this material as one of the outstanding candidates for the realization of a single material 2D CMOS technology. On the other hand, the electrostatic control on the degenerately $n$-doped $\mathrm{SnSe}_{2}$ is extremely $\operatorname{low}^{24}$. Several demonstrations of NDR in $\mathrm{WSe}_{2} / \mathrm{SnSe}_{2}$ heterojunction devices have been recently reported, but there are only two reports on n-type and p-type TFETs with minimum SS of 50 and $35 \mathrm{mV}$ per decade over one decade of drain current ${ }^{17,20}$.

Another way of achieving subthermionic SS is to benefit from the differential voltage amplification of an active gate, such as exploring the negative capacitance (NC) of ferroelectric materi$\mathrm{als}^{25}$. The negative capacitance originates from the dynamics of the stored energy in the phase transition of ferroelectric materials ${ }^{26}$. This provides an internal voltage amplification in an MOS device and hence, the channel surface potential can be changed faster than the gate voltage in an NC field-effect transistor (NC-FET) ${ }^{27,28}$. The practical implementation of the NC is difficult due to its instability. However, it is possible to partially or fully stabilize the ferroelectric capacitor in the NC state by placing it in series with a dielectric capacitor of proper value ${ }^{29}$. Until now, such NC effect has been exploited for conventional FETs ${ }^{26,30-34}$, TFETs $^{34,35}$, and 2D-FETs ${ }^{36-38}$ but never for a 2D/2D TFET. Additionally, the ferroelectric is externally connected to the gate of the conventional TFETs in the previous reported NC TFETs ${ }^{34}$. Accordingly, it would be highly desirable to explore the performance of an integrated NC 2D/2D TFET.

In this context, we have designed and experimentally characterized the NC 2D/2D TFET by incorporating the CMOS

${ }^{1}$ Nanoelectronic Devices Laboratory (NanoLab), EPFL, 1015 Lausanne, Switzerland. ${ }^{凶}$ email: sadegh.kamaeibahmaei@epfl.ch 


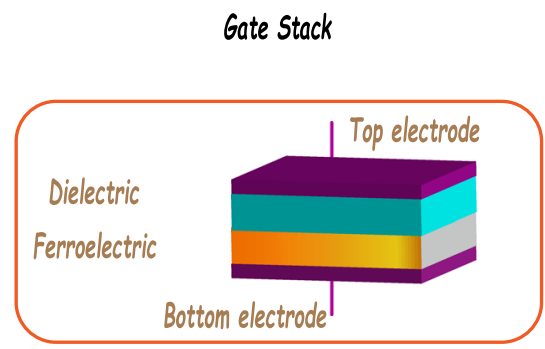

(a)

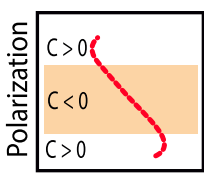

Electric field

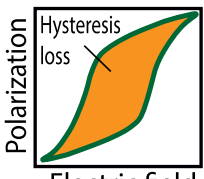

Electric field

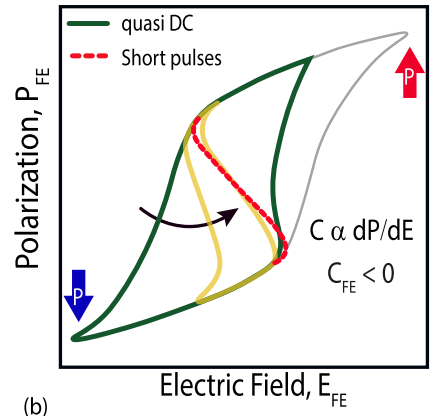

(b)
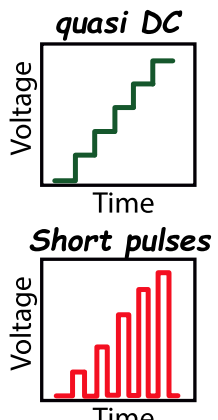

Time

Effect of temperature on ferroelectric and NC effect
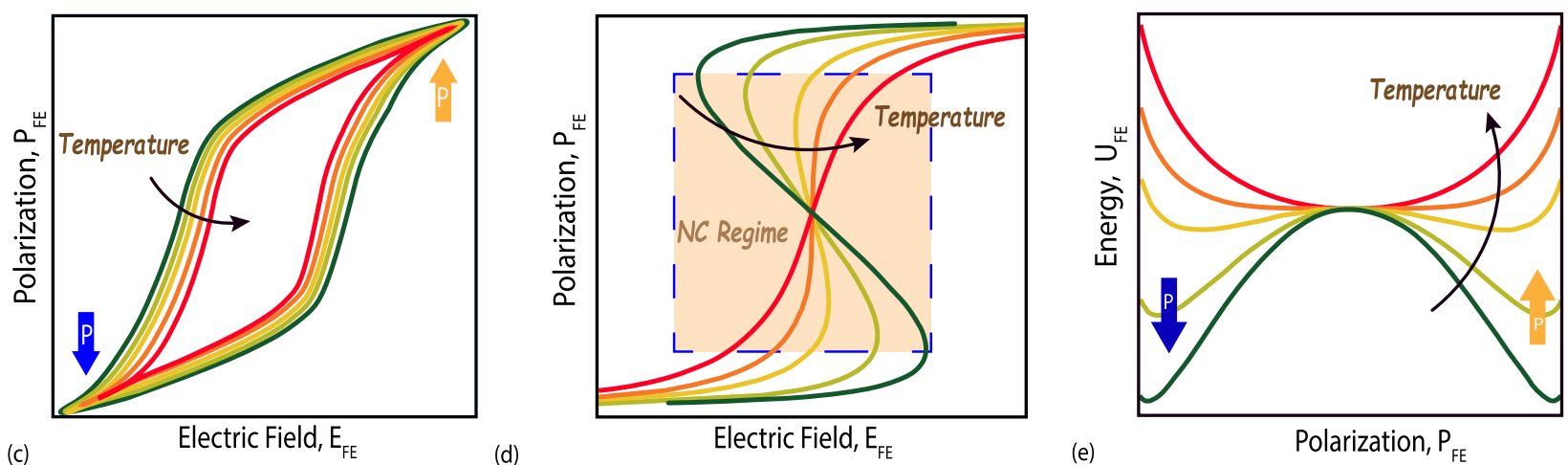

Fig. 1 Rationale and principle. a Schematic of the gate structure (left). The hysteretic behavior leads to an energy loss (right bottom) while the stable NC region without hysteresis improves the energy behavior of the gate stack (right top). b Qualitative comparison between quasiDC and pulsed measurement techniques. c Temperature-dependent $P-E$ loops. $\mathbf{d}$, e The evolution trend of the NC effect and the potential energy $U$ with increasing temperature.

compatible poly-crystalline $\mathrm{Si}: \mathrm{HfO}_{2}$ as the $\mathrm{NC}$ booster into the gate stack of a 2D/2D tunneling transistor. The bottom gated $\mathrm{WSe}_{2} /$ $\mathrm{SnSe}_{2}$ p-type TFET is fabricated based on the dry transfer technique and both the $\mathrm{WSe}_{2}$ FeFET and $\mathrm{WSe}_{2} / \mathrm{SnSe}_{2}$ FeTFET are characterized in DC mode. Gate energy efficiency factor is defined and studied at different temperatures to highlight the importance of exploiting the NC region of ferroelectrics. Additionally, this work reports a detailed study on energy charging/discharging behavior of the gate stack across a wide range of temperatures, from temperatures close to operational ones in modern microprocessors $\left(100{ }^{\circ} \mathrm{C}\right)$ down to $77 \mathrm{~K}$. The proposed device exhibits an improved SS down to $6.5 \mathrm{mV}$ per decade with a hysteresis of $1 \mathrm{~V}$ in a partially matched design, using a pulsed measurement technique. In another device structure with a fully matched design of capacitances, an average SS of $20 \mathrm{mV}$ per decade over two decades of drain current and minimum point SS less than 15 $\mathrm{mV}$ per decade are achieved with negligible hysteresis. An enhanced current efficiency factor, $g_{\mathrm{m}} / l_{\mathrm{d}}$, up to $100 \mathrm{~V}^{-1}$ is also obtained. The NC effect in 2D/2D TFETs is studied by evaluating the device performance at high temperatures, up to $100{ }^{\circ} \mathrm{C}$, in both DC and pulsed measurement techniques. The proposed device offers performance beyond the state of the art 2D/2D systems ${ }^{11,13-20}$.

A negative capacitance transistor has this property that the gate stack contributes to the differential voltage amplification and enhances the surface potential at a given gate voltage from weak to strong inversion, acting both as a subthreshold and overdrive booster ${ }^{39}$. In order to have a maximum enhancement due to the $\mathrm{NC}$ effect, the negative value of the ferroelectric's $\mathrm{NC}\left(C_{\mathrm{FE}}\right)$ should be relatively close to the intrinsic gate capacitance $\left(C_{\text {int }}\right)^{40,41}$.
Additionally, to achieve an exploitable stable regime for negative capacitance transistor in the non-hysteretic operation mode, the total capacitance of the structure should remain positive in the whole range of operation ${ }^{42}$. Note that a negative value of the total capacitance leads to instability and hysteretic behavior. It is well known that a hysteretic $I_{\mathrm{d}}-V_{\mathrm{g}}$ leads to dissipated energy $\left(E_{\mathrm{h}}\right)$, which is not favorable for energy-efficient switches (Fig. 1a).

In general, the electrostatic energy of a capacitor is given by

$\delta E=V \mathrm{~d} Q=V \frac{\mathrm{d} Q}{\mathrm{~d} t} \mathrm{~d} t=V / \mathrm{d} t$

where $V$ is the applied voltage and $Q$ is the electric charge. Similarly, the consumed energy during the charging and discharging of the gate capacitor at each point can be written as

$\delta E_{(\mathrm{i})}=V_{\mathrm{g}(\mathrm{i})} I_{\mathrm{g}(\mathrm{i})} d \mathrm{t}$

where $V_{g(i)}$ is the applied voltage and $I_{g(i)}$ is the current passing through the gate stack at the bias $V_{g(i)}$. Therefore, the energy dissipated in the gate stack due to hysteresis can be calculated as

$\delta E_{\mathrm{h}(\mathrm{i})}=\left|\delta E_{\mathrm{ch}(\mathrm{i})}\right|-\left|\delta E_{\mathrm{dis}(\mathrm{i})}\right|=\left|V_{\mathrm{g}(\mathrm{i})} I_{\mathrm{gch}(\mathrm{i})} \mathrm{d} t\right|-\left|V_{\mathrm{g}(\mathrm{i})} I_{\mathrm{gdis}(\mathrm{i})} \mathrm{d} t\right|$,

$E_{\mathrm{h}}=\sum_{i}^{N} \delta E_{\mathrm{h}(\mathrm{i})}$.

Thus, the efficiency of the gate stack can be defined as

$\eta=1-\frac{E_{\mathrm{h}}}{E_{\mathrm{ch}}+E_{\mathrm{dis}}}$,

where $E_{\mathrm{ch}}$ and $E_{\mathrm{dis}}$ are the total energy supplied during charging and discharging, respectively. As can be seen from Eq. (5), a 
hysteresis-free NC transistor has an efficiency factor of $\eta=1$ since $E_{\mathrm{h}}=0$. In another word, gate energy efficiency factor is defined to evaluate the gate control ability for the realization of steep slope switches with negligible hysteresis loss.

Figure $1 \mathrm{~b}$ qualitatively compares the impact of the pulsed and quasi-DC measurement techniques on the NC effect of ferroelectric materials. The advantage of the pulsed technique is that it applies short pulses for both biasing and measurement, which allows us to investigate the device performance before the gate leakage jeopardizes the NC effect ${ }^{43,44}$.

The differential voltage amplification of an NC booster, integrated into the gate stack of a TFET, is highly beneficial for energy band bending and enhancing the BTBT probability. This can be understood by considering the SS equation of a TFET. According to the transport mechanism by Sze and $\mathrm{Ng}^{45}$, the SS can be obtained by taking a derivative of the interband tunneling current, $l_{\mathrm{d}}=a V_{\text {eff }} \xi e^{-\frac{b}{\xi}}$

$S S=\left[\frac{\partial\left(\log l_{\mathrm{d}}\right)}{\partial V_{\mathrm{g}}}\right]^{-1}=\operatorname{Ln} 10\left[\frac{1}{V_{\text {eff }}} \frac{\partial V_{\mathrm{eff}}}{\partial V_{\mathrm{g}}}+\frac{\xi+b}{\xi^{2}} \frac{\partial \xi}{\partial V_{\mathrm{g}}}\right]^{-1}$,

where $V_{\text {eff }}$ is the tunnel-junction bias, $a$ and $b$ are the factor related to the properties of the materials constituting the heterojunction and the cross-section area $(A)$, and $\xi$ is the electric field. Accordingly, the NC effect of the incorporated ferroelectric in the gate stack of 2D/2D TFETs can modulate the channel and tunneling probability faster, providing a steeper switching. Furthermore, the derivation of $\xi$ on $V_{\mathrm{g}}$ can be boosted significantly employing NC. Supplementary Fig. 1 shows a qualitative comparison between the input transfer characteristics of a MOSFET, TFET, and NC TFET.

It is well known that the performance of a ferroelectric transistor strongly depends on the temperature ${ }^{46}$ as ferroelectric's properties are a function of temperature ${ }^{47,48}$. Figure 1c qualitatively shows the polarization hysteresis loops at different temperatures, from which the evolution of the coercive field and remnant polarization can be identified depending on the temperature. It is worth-mentioning that, depending on the field regions where the measurements are performed in, temperature scaling of the polarization hysteresis differs significantly in terms of the shape, area, remanent polarization, and coercive field ${ }^{49}$. The effects of temperature, time and electric field on polarization switching dynamics have been successfully predicted by Vopsaroiu et al. based on the classical Landau-Devonshire free energy functions in their non-equilibrium statistical mode ${ }^{50}$. For all independent switching regions (defined as elementary polar sites by the authors), the nucleation process is considered to be activated when the region of reversed polarization reaches a critical volume $V^{*}$. The reversal time of the material is determined by the switching rate of the critical volume $V^{*}$. The increased thermal energy facilitates the transition over the energy barrier, and therefore the reversal time is much faster. For our measurements with fixed frequency or electric field ramping rate, this effect is represented by a reduction of the coercive field with increasing temperature. This can be also expressed analytically based on Vopsaroiu's model ${ }^{50}$, in which the coercive field and the temperature are related by the following equation:

$E_{\mathrm{C}} \cong \frac{W_{\mathrm{B}}}{P_{\mathrm{S}}}-\frac{K_{\mathrm{B}} T}{V^{*} P_{\mathrm{S}}} \operatorname{Ln}\left(\frac{v_{0} \tau}{\ln (2)}\right)$,

where $W_{\mathrm{B}}$ is the energy barrier per unit volume, $P_{\mathrm{s}}$ is the spontaneous polarization, $v_{0}$ is the soft mode attempt frequency, $V^{*}$ is the critical nucleation activation volume, and $\tau$ is the time scale of the measurement.

Furthermore, the influence of temperature on the negativesusceptibility region of the $P-E$ curve, which is called the "S" shape behavior, is qualitatively illustrated (Fig. 1d). The NC region narrows down by increasing the temperature and almost disappears at higher temperatures. This behavior can be explained if we carefully look at the dielectric susceptibility according to the Curie-Weiss law

$X_{e}=\frac{C}{T-T_{C}}$,

where the constant of proportionality $C$ is the Curie constant and $T_{C}$ is the Curie temperature of the material where the phase transition occurs. The interest of this study is in the state of the system below $T_{\mathrm{C}}$, where the material exhibits negative susceptibility. Moreover, the relative permittivity for a ferroelectric can be approximated by $\epsilon_{\mathrm{r}}=1+X_{\mathrm{e}} \approx X \mathrm{e}$. This indicates that ferroelectric materials intrinsically exhibit negative permittivity and thus negative capacitance. Accordingly, the NC effect in ferroelectric gate stack transistors is inversely proportional to the temperature $T$.

The thermodynamic free energy density $(U)$ described within Landau-Ginzburg-Devonshire (LGD) theory ${ }^{51}$ can be expressed as a polynomial expansion up to the fourth order

$U(T)=\frac{a(T)}{2} P^{2}-\frac{\beta}{4} P^{4}-P E$,

where $\alpha$ and $\beta$ are landau coefficients. It should be noted that the coefficient $a$ represents the reciprocal of the dielectric susceptibility. Given the temperature dependency of $X_{\mathrm{e}}, a$ is considered linearly dependent on temperature while $\beta$ is temperature independent. Therefore, the energy landscape of the ferroelectric is dependent on temperature ${ }^{52}$ which is shown qualitatively in Fig. 1e.

According to the device configuration, there are multiple temperature dependencies in the transfer characteristic: ferroelectric properties, TAT, Shockley-Read-Hall (SRH), and BTBT. When the temperature decreases, the effect of SRH and TAT components is relatively minimized while the BTBT term of the current has a little dependence on the temperature. Therefore, the BTBT could prevail as the main carrier transport mechanism and hence, a relative improvement in the SS is expected in low temperatures. The enhancement of SS can be also attributed to a more effective $\mathrm{NC}$ at low temperatures. It is worth-noting that other factors such as $2 \mathrm{D} / 2 \mathrm{D}$ heterojunction area, trap density, and defect variability can also cause significant variability at room temperature. Although these variability effects can be relatively removed at our low temperature study, deep cryogenic measurement is required to completely suppress the impact of the noted parameters.

Based on Eqs. (2-5), the gate energy efficiency of a ferroelectric transistor mainly depends on hysteresis $(\Delta V)$ and gate current $\left(I_{\mathrm{g}}\right)$ during charging and discharging. As mentioned earlier in this section, the hysteresis of ferroelectric materials decreases by increasing the temperature. Therefore, it is expected that the gate energy efficiency increases at elevated temperatures. However, the other factor that plays an important role in device efficiency, the gate current, increases with the temperature which may lead to a higher energy loss. Therefore, these two effects provide two opposite trends for the gate energy efficiency versus temperature and the resulting behavior is complex and was never reported to date. The experiments reported in next sections will reveal the overall gate energy efficiency dependence on temperature from cryogenic to high temperature, in ferroelectric gate stacks using Si: $\mathrm{HfO}_{2}$ applied to a Tunneling FET device.

\section{RESULTS AND DISCUSSION}

\section{Fully integrated NC 2D/2D TFET}

The process flow designed for the fabrication of our proposed NC $\mathrm{WSe}_{2} / \mathrm{SnSe}_{2}$ TFET devices is summarized in Fig. 2a. First, a LOR/ AZ1512 bilayer was patterned by conventional photolithography to define the bottom gate contact. Then, $50 \mathrm{~nm}$ of TiN was 
(a)

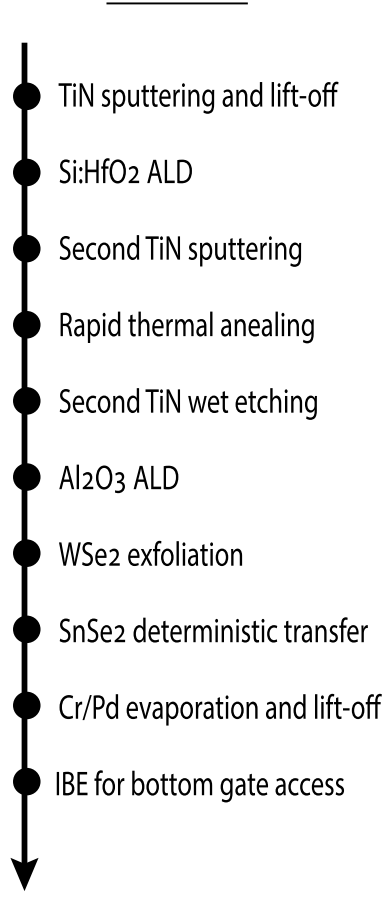

(b)

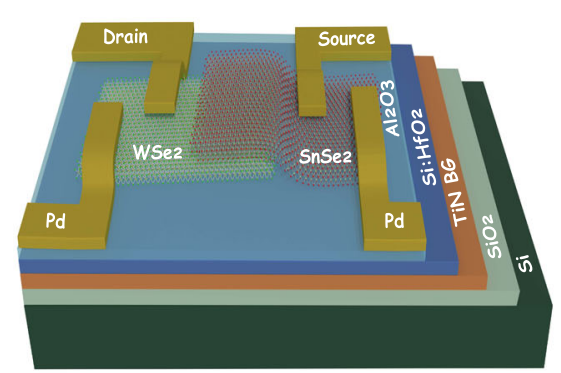

Orthorhombic phase (ferroelectric)

(d)

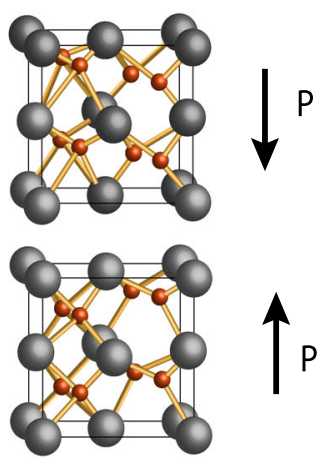

(c)

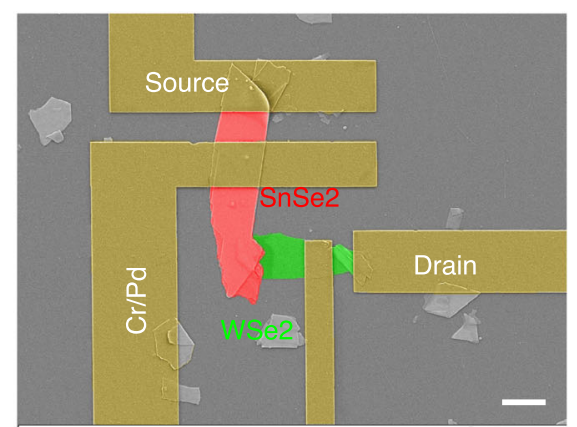

(e)

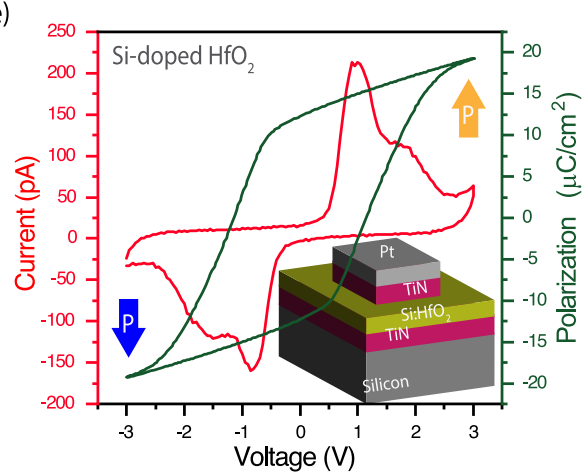

Fig. 2 Fully integrated NC 2D/2D tunnel FET. a Summary of the process flow followed for the realization of the NC WSe $2 / \mathrm{SnSe}_{2} \mathrm{TFET}$. b Schematic diagram of the $\mathrm{NC} 2 \mathrm{D} / 2 \mathrm{D}$ vdW vertical TFET in which $16 \mathrm{~nm}$ of $\mathrm{Si}_{\mathrm{HfO}}$ is integrated into the gate stack (4-nm-thick $\mathrm{Al}_{2} \mathrm{O}_{3}$ ) of a back gated $\mathrm{WSe}_{2} / \mathrm{SnSe}_{2}$ heterojunction TFET. c SEM image of the fabricated device, showing WSe $\mathrm{W}_{2}$ and $\mathrm{SnSe}_{2}$ flakes for the junction, the Pd contacts, and the overlapped tunneling region (scale bar $2 \mu \mathrm{m}$ ). d The crystal structure of $\mathrm{Si}: \mathrm{HfO}_{2}$ in its two stable polarization states, which occurs due to the ionic movement. e The $P-V$ and $I-V$ curves of a 16 -nm-thick $\mathrm{Si}_{\mathrm{HfO}} \mathrm{H}_{2}$ ferroelectric capacitor, exhibiting a sharp and coherent switching.

sputtered and lifted-off. Atomic layer deposition (ALD) of 16-nmthick Si: $\mathrm{HfO}_{2}$ followed by the sputtering of top TiN layer were performed to complete the metal-ferroelectric-metal (MFM) stack. To activate the ferroelectricity of Si: $\mathrm{HfO}_{2}$, rapid thermal annealing (RTA) in nitrogen ambient was done at $600^{\circ} \mathrm{C}$ for $2 \mathrm{~min}$. The bottom gate structure was completed by wet etching the second $\mathrm{TiN}$ layer and depositing $4 \mathrm{~nm} \mathrm{Al}_{2} \mathrm{O}_{3}$. 2D flakes of $\mathrm{WSe}_{2}$ were mechanically exfoliated directly onto the gate stack. Afterward, $\mathrm{SnSe}_{2}$ flakes were exfoliated onto a PDMS stamp stuck on a glass slide. The glass slide was used to deterministically transfer $\mathrm{SnSe}_{2}$ flakes on previously selected $\mathrm{WSe}_{2}$ flakes ${ }^{22,53}$. The electrodes of source and drain were established by a standard E-beam lithography on a MMA/PMMA bilayer resist, followed by the evaporation of $\mathrm{Cr} / \mathrm{Pd}(5 \mathrm{~nm} / 50 \mathrm{~nm})$ and lift-off process. Finally, the stack was locally etched by ion beam etching (IBE) to provide electrical access to the TiN contact.

A three dimensional schematic view of the final configuration is shown in Fig. $2 \mathrm{~b}$ which consists of $16 \mathrm{~nm}$ polycrystalline $\mathrm{Si}: \mathrm{HfO}_{2}$ and $4 \mathrm{~nm}$ amorphous $\mathrm{Al}_{2} \mathrm{O}_{3}$ layers as the gate dielectric of a WSe $/$ $\mathrm{SnSe}_{2}$ heterojunction TFET. A false-color scanning electron microscopy (SEM) image of a representative $\mathrm{NC} \mathrm{WSe_{2 }} / \mathrm{SnSe}_{2}$ TFET is illustrated in Fig. 2c. The different flakes, the heterojunction region, and the metallic contacts are visible in this image. The present devices are fabricated on 8-nm-thick $\mathrm{WSe}_{2}$ flakes and relatively thick $\mathrm{SnSe}_{2}$ flakes (larger than 50-nm-thick), as confirmed by the atomic force microscopy (AFM) profile shown in Supplementary Fig. 2. Figure $2 d$ is the crystal structure of two stable polarization states of $\mathrm{Si}: \mathrm{HfO}_{2}$. The MFM capacitor used in this work is characterized by measuring both the $I-V$ and polarization hysteresis loops to ensure ferroelectric behavior (Fig. 2e). A sharp and coherent domain switching is observed and further validated by piezoelectric force microscopy (PFM) (Supplementary Fig. 2).

\section{Characterization}

The electrical properties of individual $\mathrm{WSe}_{2}$ and $\mathrm{SnSe}_{2}$ flakes have been characterized at room temperature to confirm the electrical polarity of these two kinds of semiconductor. The DC characteristics of both $\mathrm{SnSe}_{2}$ and WSe $\mathrm{WeFETs}_{2}$ are fully demonstrated in the Supplementary Figs. 3 and 4. The electrical characteristics of the WSe $\mathrm{SnSe}_{2}$ heterostructure FeTFET fabricated on the same flake of $\mathrm{WSe}_{2}$ FeFET are also shown in Supplementary Fig. 5. The input transfer curve of the fabricated device exhibits the p-type tunneling transistor behavior at RT. Comparing the DC characteristics of the proposed $\mathrm{WSe}_{2} / \mathrm{SnSe}_{2}$ heterostructure FeTFET and $\mathrm{WSe}_{2}$ FeFET confirm that the main charge injection mechanism in our 2D/2D device is BTBT of carriers ${ }^{20}$. A reference 2D/2D TFET with a 10-nm thick $\mathrm{HfO}_{2}$ gate has been fabricated to evaluate the impact of ferroelectric in the gate stack of a 2D/2D system. The device is fully characterized and the results are reported in the Supplementary section.

\section{Energy charging and discharging at cryogenic temperatures}

The energy charging and discharging behavior of the fabricated ferroelectric gate stack has been investigated by decreasing the temperature down to $77 \mathrm{~K}$ (liquid nitrogen). Figure 3a shows the double sweep transfer characteristics, $l_{\mathrm{d}}-V_{\mathrm{g}}$, at different temperatures for a drain voltage $V_{d}$ of $1 \mathrm{~V}(2 \mathrm{D} / 2 \mathrm{D}$ FeTFET\#1). The extracted hysteresis values at different temperatures are shown in the inset of Fig. 3a. The hysteresis is effectively reduced when the temperature decreases down to $77 \mathrm{~K}$. The smaller hysteresis for lower temperature is possibly related to the larger coercive field of 

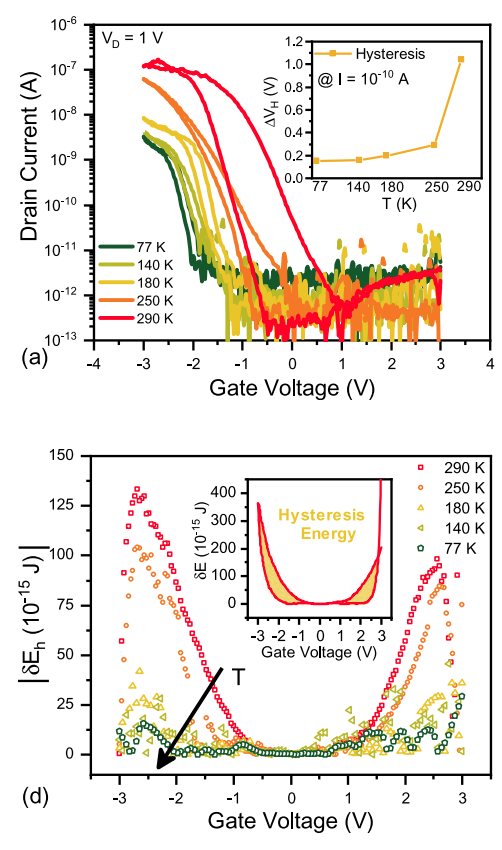
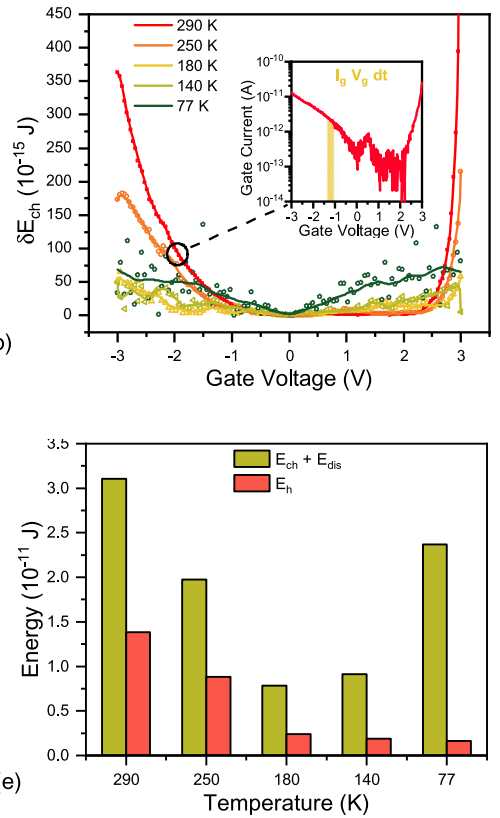

(c)
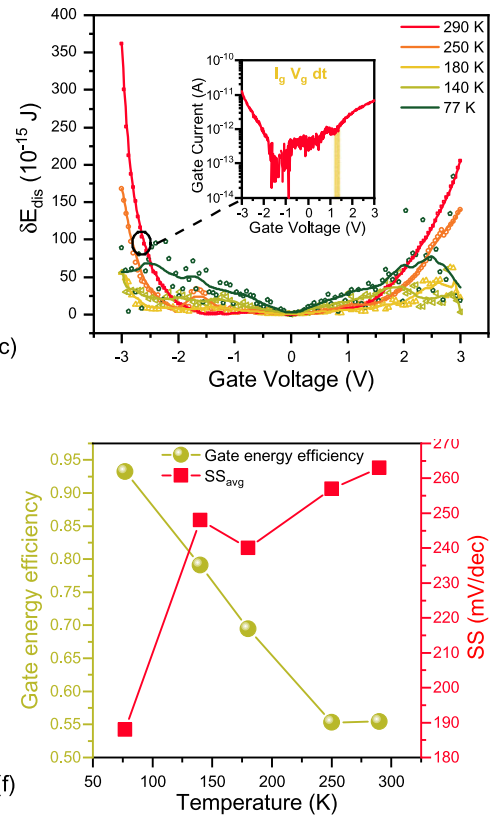
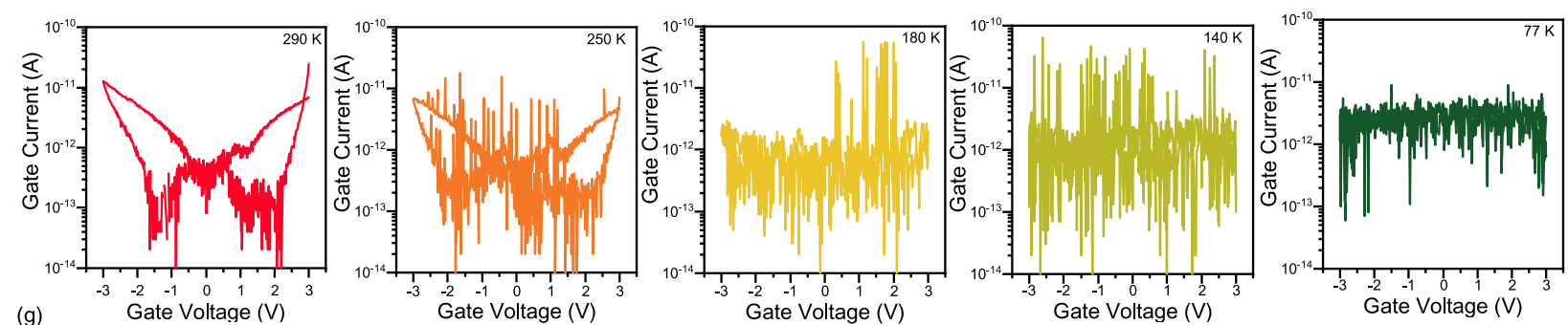

Fig. 3 Electrical and energy behavior characteristics of the 2D/2D FeTFET at low temperatures. a Performance of the device at low temperatures, down to $77 \mathrm{~K}$, while the $V_{\mathrm{d}}$ is set to $1 \mathrm{~V}$. The extracted point and average subthreshold swings show a little dependence on the temperature (Inset figure). b, c Charging and discharging energy behavior of the gate stack and $\mathbf{d}$ corresponding energy loss at low temperatures $\left(V_{d}=1 \mathrm{~V}\right)$. e Evolution of the hysteresis energy and total supplied energy with decreasing temperature. $\mathbf{f}$ Extracted average subthreshold swing and gate energy efficiency factor at different temperatures. $\mathbf{g}$ Gate current vs gate voltage at different temperatures.

the ferroelectric, and to the beneficially effect of low temperatures to thermally de-activate traps at the interface between $\mathrm{WSe}_{2}$ and the back gate and the $\mathrm{WSe}_{2} / \mathrm{SnSe}_{2}$ interface. The direct comparison between the transfer characteristics, SS as a function of the drain current and output characteristics of the device for both the 77 and $290 \mathrm{~K}$ temperatures are shown in Supplementary Fig. 7. Additionally, the output characteristic of the device is studied to further validate the BTBT transportation mechanism in our device. A negative differential resistance effect, NDR, is observed at $77 \mathrm{~K}$, which fully confirms the band-to-band tunneling of carriers in a $\mathrm{WSe}_{2} / \mathrm{SnSe}_{2}$ heterostructure tunnel FET device (Supplementary Fig. 8). The device performance at $77 \mathrm{~K}$ is reported in Supplementary Fig. 8, showing the resulting improvement at cryogenic temperatures as well as the robustness of our proposed devise at low temperatures.

The energy charging and discharging behavior of the gate stack in forward and reverse sweeps of the gate voltage at the investigated temperatures are studied. The energy plots are obtained according to Eq. (2) and based on the forward and reverse current of the gate shown in Fig. $3 \mathrm{~g}$. The extracted energy during charging $\left(\delta E_{\mathrm{ch}}\right)$ and discharging $\left(\delta E_{\mathrm{dis}}\right)$ as a function of the gate voltage are demonstrated in Fig. $3 b$, c, while the hysteresis energy $\left(\delta E_{\mathrm{h}}\right)$ is highlighted in Fig. 3d. Figure 3e illustrates the total energy during charging/discharging and the dissipated energy due to hysteresis $\left(E_{\mathrm{h}}\right)$ for all the temperatures. Lower hysteresis energy is observed with the decrease of the temperature. This can be explained by considering the fact that due to the larger coercive field and multi-domain structure of $\mathrm{Si}: \mathrm{HfO}_{2}$, only a small fraction of the polarization gets switched for the investigated range of voltages at low temperatures, providing a relatively smaller hysteresis. On the other hand, at cryogenic temperatures, the slope of the S-shape characteristics offers better conditions for a larger stable range of NC, which would result in non-hysteretic $l_{\mathrm{d}}-V_{\mathrm{g}}$ characteristics. Therefore, overall a much lower energy loss is expected at cryogenic temperatures, as confirmed by our experimental results. Additionally, the total charging and discharging energies during forward and reverse sweeps is decreasing with temperature and it starts to increase above $180 \mathrm{~K}$. We believe that the increase of the charging/discharging energy below $180 \mathrm{~K}$ is due to the fact that the gate current reduces below the detection limit of our measurement setup, and cannot be distinguished by the noise. Therefore, a measurement system with higher precision would confirm that the charge/discharge energy decreases monotonically by reducing the temperature. The gate energy efficiency factor and average value of SS with respect to the studied temperatures are shown in Fig. 3f. The continuous yet still limited improvement of SS with temperature indicates that the trap-assisted tunneling (TAT) may remain a significant contribution to the charge transport, and lower temperatures are needed to fully remove it to significantly improve the slope ${ }^{54,55}$. Such an assumption is also consistent that even an improved NC effect at a lower temperature will have little effect 

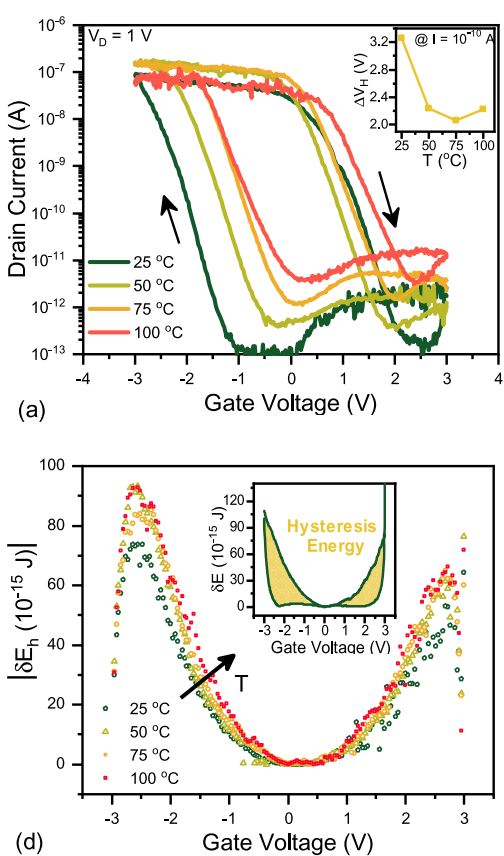

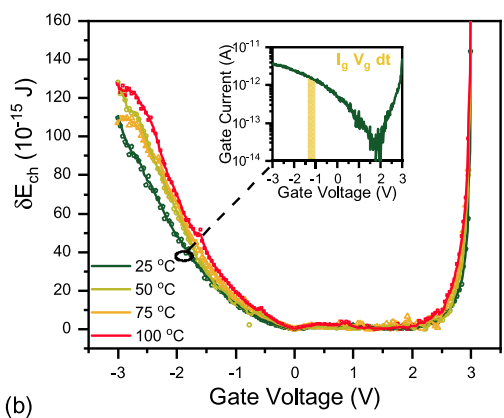

(b)

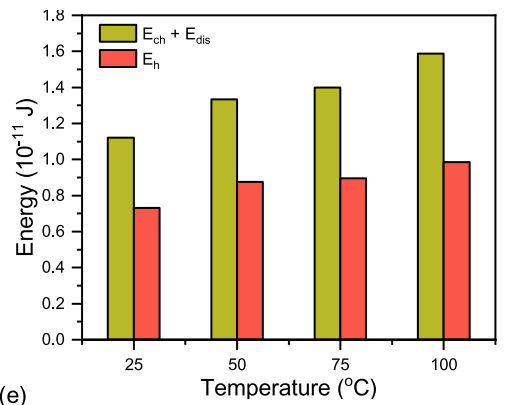

(c)
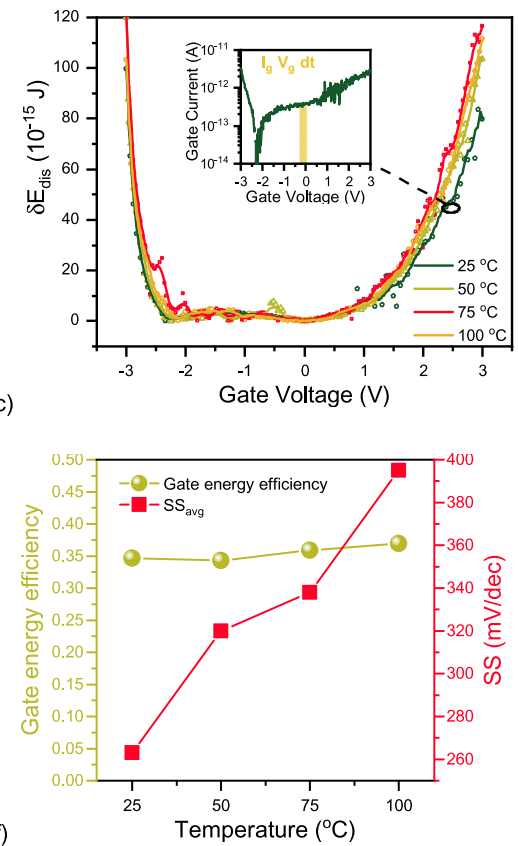

Fig. 4 Electrical and energy behavior characteristics of the 2D/2D FeTFET at high temperatures. a Input transfer characteristics of the 2D/ 2D FeTFET at $V_{\mathbf{d}}=1 \mathrm{~V}$ for different temperatures. b, c Energy behavior of the FeTFET gate stack in forward and reverse sweeps of $V_{\mathrm{g}}$. $\mathbf{d}$ Energy loss of the gate capacitors during a dual sweep of the device. e The total energy consumed during charging/discharging and the energy dissipated due to hysteresis at elevated temperatures. f The gate energy efficiency factor and SS with respect to the investigated temperatures.

on a TAT current contribution. In our device, the experimental average and best point swings are near 188 and $69 \mathrm{mV}$ per decade at $77 \mathrm{~K}$, respectively, while these values reach 263 and 153 $\mathrm{mV}$ per decade at $290 \mathrm{~K}$. A higher $\eta$ is observed at lower temperatures due to the reduction of the hysteresis and hence, the lower dissipated energy. The 2D/2D FeTFET has an efficiency factor of $93 \%$ and $55 \%$ at $77 \mathrm{~K}$ and $290 \mathrm{~K}$, respectively.

\section{Energy charging and discharging at high temperatures}

In order to further elaborate on the gate energy behavior, another series of experiments have been conducted on the 2D/2D FeTFET\#1 at high temperatures up to $100{ }^{\circ} \mathrm{C}$. Figure $4 a$ shows the transfer characteristics, $l_{\mathrm{d}}-V_{\mathrm{g}}$, from room temperature up to $100{ }^{\circ} \mathrm{C}$, with the corresponding hysteresis values reported in the inset figure. The higher $I_{\text {off }}$ corresponds to the temperature dependence of the SRH leakage current, and is responsible for the apparent deterioration of the point and average subthreshold slopes. A relatively smaller hysteresis is observed at elevated temperatures. This can be explained by the increase of the leakage current and temperature dependency of the thermal coefficient and the coercive field of the ferroelectric material. These results demonstrate that the ferroelectricity is well preserved in the Si: $\mathrm{HfO}_{2}$ stack at elevated temperatures close to the working temperature of modern processors.

To have a more comprehensive understanding of the ferroelectric gate stack behavior as a function of temperature, the charging and discharging energies of the gate capacitor are calculated based on gate leakage current when performing forward and reverse gate voltage sweeps, as shown in Supplementary Fig. 9. We observe a clear butterfly shape in the gate leakage current characteristics for all the investigated temperatures, which is related to the switching behavior of the ferroelectric in the gate stack. The energy of the gate capacitor during charging $\left(\delta E_{\mathrm{ch}}\right)$, discharging $\left(\delta E_{\mathrm{dis}}\right)$ and the absolute value of their difference $\left(\delta E_{\mathrm{h}}\right)$ are shown in Fig. $4 \mathrm{~b}-\mathrm{d}$. For each temperature, the energy plots are calculated according to Eqs. (2) and (3). The total energy during the charging/discharging and the energy of hysteresis at different temperatures are presented in Fig. 4e. It is evidenced that both the hysteresis energy and charging/discharging energy are increased by the temperature increase. This is due to the fact that the impact of the increase of the leakage current is dominant compared to the hysteresis. Gate energy efficiency factor and average subthreshold swing are illustrated in Fig. 4f, at temperatures close to the operational one in microprocessors. In a tunnel FET where the main conduction is based on BTBT, one would expect a little dependence of the subthreshold swing on the temperature. However, in the low current range, the SS of the ferroelectric gate Tunnel FET is relatively deteriorated, as shown in Fig. $4 \mathrm{f}$, essentially due to the influence of the SRH leakage increase at elevated temperatures and also, possibly due to the temperature dependence negative slope of the S-shape polarization characteristics. On the other hand, the gate energy efficiency factor remains almost constant, which suggest that in the range of studied temperature (up to $100^{\circ} \mathrm{C}$ ), far from the critical Tc of the investigated gate stack, the proposed ferroelectric Tunnel FET transistor will not have significant gate energy efficiency deterioration. This is due to the fact that the gate current and hysteresis, two important factors in gate efficiency, provide opposite trends at elevated temperatures, as explained earlier. Additionally, it should be noted that the relatively small difference in the gate energy efficiency value at $300 \mathrm{~K}$ in Figs. $4 \mathrm{f}$ and $3 \mathrm{f}$ is attributed to the fact that the data in Fig. $4 \mathrm{f}$ has been extracted after the cooling of the sample down to $77 \mathrm{~K}$. Therefore, the interface traps between flake and the linear dielectric, the $\mathrm{SnSe}_{2} / \mathrm{WSe}_{2}$ interface and the source and drain contacts on the flakes are going to be relatively modified. Given the gate energy efficiency factor formula, any changes in the hysteresis can affect the gate energy efficiency value. Moreover, the measurement under vacuum could be another contributing 

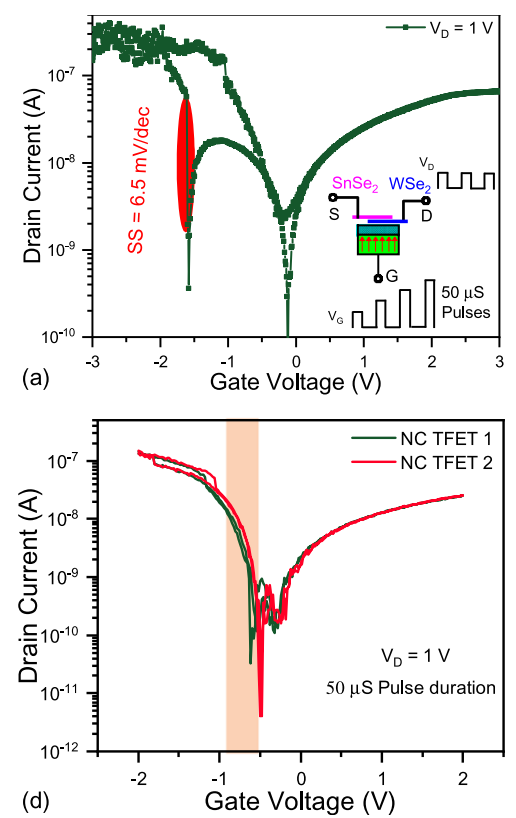

(b)
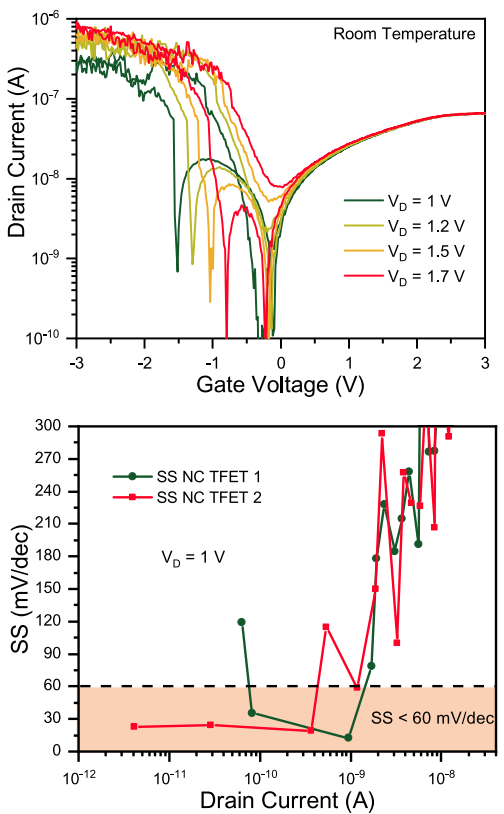

(c)
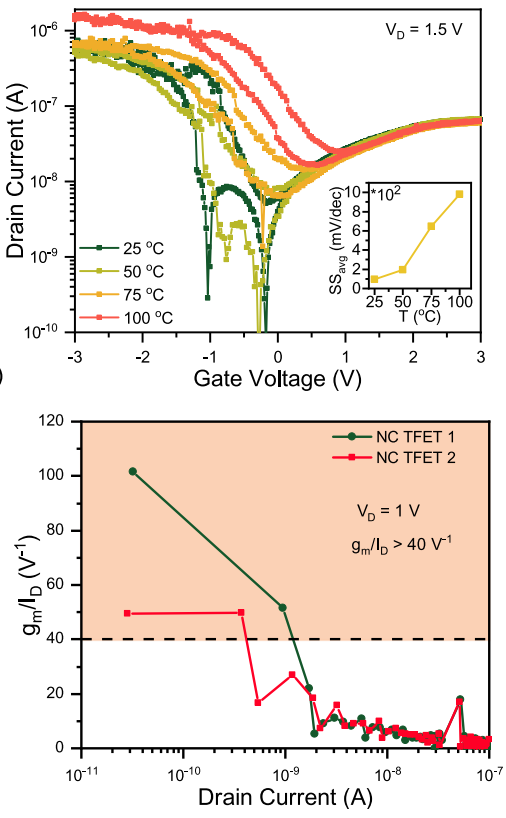

Fig. 5 Pulsed measurements of the hysteretic and hysteresis-free NC 2D/2D TFETs. a $l_{d}-V_{g}$ curve of the NC-TFET, showing a steep SS of 6.5 $\mathrm{mV}$ per decade over almost two decades of current. The employed pulsed measurement technique is schematically demonstrated in the inset figure. $\mathbf{b}$ Transfer characteristic of the NC-TFET at multiple drain voltages, showing a clear degradation of SS by increasing $V_{\mathrm{d}}$. $\mathbf{c} l_{\mathrm{d}}-V_{\mathrm{g}}$ plots of the same device in different temperatures $\left(V_{d}=1.5 \mathrm{~V}\right)$. The SS of the device increases at elevated temperatures. $\mathbf{d}$ Transfer characteristic of two different non-hysteretic NC-TFETs, and e the corresponding subthreshold swings. A sub-thermionic SS, down to $15 \mathrm{mV}$ per decade over two decades of current, is reported at room temperature. $f$ Transconductance efficiency of both devices, showing a peak of $50 \mathrm{~V}^{-1}$ and 100 $\mathrm{V}^{-1}$. The peak transconductance efficiency exceeds the fundamental $40 \mathrm{~V}^{-1}$ limit of MOSFET devices.

factor to this relatively small difference. In general, these results show that considerable energy is lost in each cycle when the ferroelectric operates in the hysteretic mode.Therefore, a significant improvement in efficiency can be achieved if the ferroelectric performs a true hysteresis-free NC effect.

\section{Pulsed measurement}

Pulsed measurement technique have been proposed as a valuable tool for the exploration and demonstration of NC in ferroelectric materials, due to its ability to reduce charge injection assisted switching and ferroelectric's leakage current ${ }^{44,56}$. Therefore, to demonstrate that in our fabricated devices can reach the NC regime, we have carried out our experiments on the pulsed mode characterization of NC 2D/2D TFET. To experimentally investigate this structure in which the NC effect is combined with a 2D/2D TFET, short pulses were applied to the gate electrode. The device configuration and measurement setup can be found in the Supplementary. Additionally, the impact of the pulse duration/ frequency is fully investigated and discussed in the Supplementary. Based on the obtained results, a pulse duration on the order of $90 \mu \mathrm{s}$ is used to extract the transfer characteristics. The reported current in the $I_{\mathrm{d}}-V_{\mathrm{g}}$ plots is an average of readings taken in a predefined measurement window of each pulse. This window is after the rising and before the falling of the applied pulses on the gate.

\section{Hysteretic NC 2D/2D TFET}

In this section, the electrical performance of a hysteretic NC 2D/2D TFET, where the NC of ferroelectric is partially stabilized, is reported. It should be noted that the device used for this experiment is the same as previous section, 2D/2D FeFET\#1. Figure 5 a illustrates the $I_{\mathrm{d}}-V_{\mathrm{g}}$ plot of the NC-TFET, where the gate voltage is swept from $3 \mathrm{~V}$ to $-3 \mathrm{~V}$ and back to $3 \mathrm{~V}$ while the drain voltage is set at $1 \mathrm{~V}$. The inset shows the schematic of the pulsed measurement technique. The DC characteristics of this device were previously demonstrated in Fig. $4 a$. An improvement in the subthreshold swing is explicitly observed due to the differential voltage amplification. A measured point SS of $6.5 \mathrm{mV}$ per decade and an average SS of $15 \mathrm{mV}$ per decade over almost two decades of the drain current is achieved at RT. A relatively large hysteresis of $1 \mathrm{~V}$ is observed due to the fact that the $\mathrm{NC}$ of $\mathrm{Si}: \mathrm{HfO}_{2}$ is stabilized only in a limited range of the gate voltage ${ }^{42,57}$. The transfer characteristic of the NC 2D/2D TFET is also measured at different drain voltages varied from 1 to $1.7 \mathrm{~V}$, while the pulse duration is constant (Fig. 5b). It is evidenced that the steepness of the subthreshold swing can be affected by the variation of the drain voltage as the operation point of an NC transistor depends also on the drain bias ${ }^{40}$. The impact of the temperature on the performance of our proposed structure is also investigated by measuring and analyzing the $I_{\mathrm{d}}-V_{\mathrm{g}}$ curves at multiple temperatures with a constant voltage of $1.5 \mathrm{~V}$ (Fig. $5 \mathrm{C}$ ). Inset of this figure exhibits how the extracted average of SS is deteriorated when the temperature increases, showing the impact of temperature on the value of ferroelectric NC. Supplementary Fig. 14 reports the $I_{\mathrm{d}}-V_{\mathrm{g}}$ plots at different temperatures when $V_{d}$ is set at $1.7 \mathrm{~V}$. Although, the ferroelectric capacitor in this structure implies an NC effect, a large hysteresis together with a sharp transition is not appealing for transistor applications. The hysteresis of the NC 2D/2D TFET can be alleviated by modifying the value of the ferroelectric NC, and the effective gate capacitance of TFET to ensure a fully matched design of capacitances in the whole range of operation.

\section{Hysteresis-free NC 2D/2D TFET}

Here, other NC 2D/2D TFETs with the same configuration and the NC matching condition close to the ideal are presented. A negligible hysteresis of less than $10 \mathrm{mV}$ is obtained for two TFETs designed on the same chip (Fig. 5d). SEM images and DC 
characteristics of the fabricated devices for this experiment, NC TFET\#1 and NC TFET\#2, are depicted in Supplementary Figs. 15 and 16 , respectively. The gate voltage was swept from 2 to $-2 \mathrm{~V}$ and back to the initial point with the same pulse condition and drain voltage of the previous experiment. It is expected that any changes in the pulse frequency can affect both the SS and hysteresis of the transfer characteristic, as experimentally demonstrated in the Supplementary information. Figure $5 e$ shows the subthreshold swing as a function of the output current for both of the investigated NC TFETs, exhibiting a steep off-to-on transition. The minimum point subthreshold slope of $15 \mathrm{mV}$ per decade and an average of $30 \mathrm{mV}$ per decade over 1 order of drain current is reported for the NC TFET\#1 at RT. In NC TFET\#2, the SS falls below the Boltzman limit for over two decades of current, with an average subthreshold swing of $20 \mathrm{mV}$ per decade at RT. These results confirm that a hysteresis-free NC-TFET, suitable for logic applications, can be realized by properly satisfying the NC matching condition ${ }^{57}$. Although, the gate stack is similar for all the reported devices, the capacitance per unit area is different and defined by the overlap between the drain and source electrode, and the geometry of the flakes. Therefore, different gate capacitances and NC effects are expected. The larger SS and smaller hysteresis in these cases compared to the previous experiment are probably due to the negative capacitance value in the investigated range of the gate voltage. This means that a trade-off is required between the hysteretic behavior and the performance-boosting that is caused by the NC of the ferroelectric ${ }^{58}$. In these devices, the total capacitance of the structure remains positive in a wider range of the $V_{\mathrm{g}}$ which leads to a nonhysteretic transfer characteristic. Further characterization of these NC 2D/2D TFETs revealed an abrupt and dramatic switching behavior of the current efficiency factor, $g_{\mathrm{m}} / I_{\mathrm{d}}$, as an analog figure of merit of transistors ${ }^{59}$. The extracted values are reported in Fig. $5 \mathrm{f}$ which demonstrates peaks of 100 and $50 \mathrm{~V}^{-1}$ over one decade of drain current for NC TFET\#1 and \#2, respectively. These values exceed the $40 \mathrm{~V}^{-1}$ analog efficiency limit of MOSFET, highlighting the effect of integrating a ferroelectric layer into the gate stack of a 2D/2D TFET. Supplementary table 1 summarizes the performance of the NC 2D/2D TFET. The lowest point SS, sub-60 mV per decade $\mathrm{SS}_{\mathrm{avg}}$ over two decades of the drain current, negligible hysteresis, with reasonable ON/OFF current ratio and the possibility of integrating the NC and heterojunction TFET are achieved. A well-designed negative capacitor integrated to the gate stack of a 2D/2D TFET significantly improved the SS and hysteresis. Therefore, an NC $2 \mathrm{D} / 2 \mathrm{D}$ platform is a potential configuration for energy-efficient applications.

\section{DISCUSSION}

We have reported a comprehensive experimental study of a NC Si: $\mathrm{HfO}_{2}$ ferroelectric gate stack WSe $/ \mathrm{SnSe}_{2}$ TFET focused on the influence of the temperature gate charging and discharging and static characteristics. A gate energy efficiency factor has been defined and used to evaluate the energy efficiency of the gate stack. The energy behavior in ferroelectric gate stack is studied using DC measurement techniques in a wide range of temperatures, from cryogenic temperature, $77 \mathrm{~K}$, to $100^{\circ} \mathrm{C}$, to highlight the importance of exploiting the non-hysteretic NC regime. A pulsed measurement technique has been employed to enable the visualization of NC effect on the device characteristics. In a partially matched NC capacitor, a hysteretic NC effect leads to an SS of 6.5 $\mathrm{mV}$ per decade with an average value of $15 \mathrm{mV}$ per decade. In another configuration, near hysteresis-free NC 2D/2D TFETs with point sub-thermionic swing below $15 \mathrm{mV}$ per decade, average swings of $15-20 \mathrm{mV}$ per decade over almost two decades of current and ON/OFF current ratio exceeding $10^{4}$ are demonstrated. An enhanced current efficiency factor up to $100 \mathrm{~V}^{-1}$ is reported, presenting a well-designed $\mathrm{NC}$ as an analog booster for 2D/2D
TFETs. We explored NC 2D/2D TFET performances from cryogenic temperature up to $100{ }^{\circ} \mathrm{C}$, showing device reproducibility and robustness. The experimentally reported results contribute to the performance engineering of 2D/2D systems for the realization of high performance steep-slope devices. This study provides an insight into the potential of a negative capacitance booster to address the most limiting performances of 2D/2D TFETs. Additionally, the energy behavior of the ferroelectric gate stack under NC condition fulfillment can be effectively applied as a universal method to design more energy efficient switches.

\section{METHODS}

\section{Si: $\mathrm{HfO}_{2}$ deposition}

Sixteen nanometer of silicon-doped $\mathrm{HfO}_{2}$ were deposited by atomic layer deposition (ALD) in a BENEQ TFS200 ALD tool. The starting substrate was a p-doped $\mathrm{Si} /(100 \mathrm{~nm}) \mathrm{SiO}_{2}$ wafer covered by $50 \mathrm{~nm}$ TiN as the bottom electrode. The precursors were tetrakis(ethylmethylamino)hafnium (TEMA-Hf) for $\mathrm{Hf}$ and $\mathrm{SiH}_{2}\left(\mathrm{~N}_{2}\left(\mathrm{C}_{2} \mathrm{H}_{5}\right)_{2}\right)_{2}$ (SAM24) for $\mathrm{SiO}_{2}$. Water and ozone were used as the oxygen source and the deposition temperature was $300{ }^{\circ} \mathrm{C}$. The $\mathrm{Si}: \mathrm{HfO}_{2}$ film was achieved by alternating ALD cycles of TEMA-Hf and SAM24. The cycle ratio of 16:1:16 $\left(\mathrm{HfO}_{2}: \mathrm{SiO}_{2}\right.$ : $\mathrm{HfO}_{2}$ ) corresponds to a silicon concentration of $3.4 \%$ and a thickness of 13-16 nm was used in this experiment. Top TiN of $10 \mathrm{~nm}$ was then deposited by sputtering of TiN target in an Alliance-Concept DP650 physical vapor deposition tool at room temperature. Rapid thermal annealing (RTA) in nitrogen ambient was performed for 2 min at $600{ }^{\circ} \mathrm{C}$ to activate the ferroelectricity and complete the metal-ferroelectricmetal (MFM) structure.

\section{TiN wet etching}

Top TiN electrode was etched away after the RTA step using the wet etch process. The sample was placed in a solution consists of $10 \mathrm{ml} \mathrm{NH}_{4} \mathrm{OH}, 20$ $\mathrm{ml} \mathrm{H}_{2} \mathrm{O}_{2}$, and $500 \mathrm{ml} \mathrm{H} 2 \mathrm{O}$ solution at $50{ }^{\circ} \mathrm{C}$ for $3 \mathrm{~min}$.

\section{Characterization}

The measurement of electrical properties in DC mode was acquired with a conventional semiconductor parameter analyzer and electrical probes. In all the reported electrical measurements the $\mathrm{WSe}_{2}$ is biased as the drain of the device. For pulsed measurements, the pulses were applied to the gate by a Keithley 4200A-SCS with 4225-PMU and simultaneously, the output current is measured. Rise and fall time of the voltage pulses were fixed to $15 \mu \mathrm{s}$ each. The duration was set to $90 \mu \mathrm{s}$ for each pulse.

\section{Metrology}

SEM analysis of the devices was carried out on a Zeiss Merlin SEM. AFM in contact mode and the off-resonance PFM were performed by using Cypher AFM (Asylum Research) for accurate thickness estimation and switching behavior of Si:HfO electric field were performed using an AixACCT TF 2000 tester with an FE module.

\section{DATA AVAILABILITY}

The raw data used in this study are available upon reasonable request to the corresponding author.

Received: 4 March 2021; Accepted: 10 August 2021; Published online: 07 September 2021

\section{REFERENCES}

1. Takagi, S. et al. Carrier-transport-enhanced channel CMOS for improved power consumption and performance. IEEE Trans. Electron Devices 55, 21-39 (2007).

2. Yeric, G. Moore's law at 50: are we planning for retirement? In 2015 IEEE International Electron Devices Meeting (IEDM) 1-1 (IEEE, 2015).

3. Meindl, J. D. Beyond Moore's law: the interconnect era. Comput. Sci. Eng. 5, 20-24 (2003). 
4. Ionescu, A. M. \& Riel, H. Tunnel field-effect transistors as energy-efficient electronic switches. Nature 479, 329-337 (2011).

5. Shukla, N. et al. A steep-slope transistor based on abrupt electronic phase transition. Nat. Commun. 6, 1-6 (2015).

6. McGuire, F. A., Cheng, Z., Price, K. \& Franklin, A. D. Sub-60 mV/decade switching in 2D negative capacitance field-effect transistors with integrated ferroelectric polymer. Appl. Phys. Lett. 109, 093101 (2016).

7. Memisevic, E. et al. Individual defects in $\ln \mathrm{As} / \mathrm{InGaAsSb} / \mathrm{GaSb}$ nanowire tunnel field-effect transistors operating below $60 \mathrm{mV} /$ decade. Nano Lett. 17, 4373-4380 (2017).

8. Agarwal, S. \& Yablonovitch, E. Band-edge steepness obtained from Esaki/backward diode current-voltage characteristics. IEEE Trans. Electron Devices 61, 1488-1493 (2014).

9. Ganjipour, B., Wallentin, J., Borgstrom, M. T., Samuelson, L. \& Thelander, C. Tunnel field-effect transistors based on InP-GaAs heterostructure nanowires. ACS Nano 6 , 3109-3113 (2012).

10. Novoselov, K., Mishchenko, O. A., Carvalho, O. A. \& Neto, A. C. 2D materials and van der Waals heterostructures. Science 353, aac9439 (2016).

11. Fan, S. et al. Tunable negative differential resistance in van der Waals heterostructures at room temperature by tailoring the interface. ACS Nano 13, 8193-8201 (2019).

12. Roy, T. et al. Dual-gated $\mathrm{MoS}_{2} / \mathrm{WSe}_{2}$ van der Waals tunnel diodes and transistors. ACS Nano 9, 2071-2079 (2015).

13. Nourbakhsh, A., Zubair, A., Dresselhaus, M. S. \& Palacios, T. Transport properties of a $\mathrm{MoS}_{2} / \mathrm{WSe}_{2}$ heterojunction transistor and its potential for application. Nano Lett. 16, 1359-1366 (2016).

14. Guo, Z. et al. Independent band modulation in $2 \mathrm{D}$ van der Waals heterostructures via a novel device architecture. Adv. Sci. 5, 1800237 (2018).

15. He, J. et al. 2D tunnel field effect transistors (FETs) with a stable charge-transfertype $\mathrm{p}+-\mathrm{WSe}_{2}$ source. Adv. Electron. Mater. 4, 1800207 (2018).

16. Xu, J., Jia, J., Lai, S., Ju, J. \& Lee, S. Tunneling field effect transistor integrated with black phosphorus-MoS ${ }_{2}$ junction and ion gel dielectric. Appl. Phys. Lett. 110, 033103 (2017).

17. Yan, X. et al. Tunable $\mathrm{SnSe}_{2} / \mathrm{WSe}_{2}$ heterostructure tunneling field effect transistor Small 13, 1701478 (2017).

18. Sarkar, D. et al. A subthermionic tunnel field-effect transistor with an atomically thin channel. Nature 526, 91-95 (2015).

19. Roy, T. et al. 2D-2D tunneling field-effect transistors using $\mathrm{WSe}_{2} / \mathrm{SnSe}_{2}$ heterostructures. Appl. Phys. Lett. 108, 083111 (2016).

20. Oliva, N. et al. WSe $e_{2} / \mathrm{SnSe}_{2}$ vdW heterojunction tunnel FET with subthermionic characteristic and MOSFET co-integrated on same WSe $\mathrm{W}_{2}$ flake. npj 2D Mater. Appl. 4, 1-8 (2020).

21. Resta, G. V. et al. Polarity control in $\mathrm{WSe}_{2}$ double-gate transistors. Sci. Rep. 6 , 29448 (2016).

22. Kamaei, S. et al. An experimental study on mixed-dimensional 1D-2D van der Waals single-walled carbon nanotube-WSe ${ }_{2}$ hetero-junction. IEEE Electron Device Lett. 41, 645-648 (2020).

23. Allain, A. \& Kis, A. Electron and hole mobilities in single-layer WSe $\mathrm{W}_{2}$. ACS Nano 8 , 7180-7185 (2014).

24. Guo, C., Tian, Z., Xiao, Y., Mi, Q. \& Xue, J. Field-effect transistors of high-mobility few-layer SnSe 2 . Appl. Phys. Lett. 109, 203104 (2016)

25. Salahuddin, S. \& Datta, S. Use of negative capacitance to provide voltage amplification for low power nanoscale devices. Nano Lett. 8, 405-410 (2008).

26. Zubko, P. et al. Negative capacitance in multidomain ferroelectric superlattices Nature 534, 524-528 (2016)

27. Ionescu, A. M. Negative capacitance gives a positive boost. Nat. Nanotechnol. 13 , 7-8 (2018).

28. Gao, W. et al. Room-temperature negative capacitance in a ferroelectric-dielectric superlattice heterostructure. Nano Lett. 14, 5814-5819 (2014).

29. Appleby, D. J. et al. Experimental observation of negative capacitance in ferroelectrics at room temperature. Nano Lett. 14, 3864-3868 (2014).

30. Li, K.-S. et al. Sub-60mV-swing negative-capacitance FinFET without hysteresis. In 2015 IEEE International Electron Devices Meeting (IEDM) 22-6 (IEEE, 2015).

31. Lee, M. et al. Prospects for ferroelectric HfZrOx FETs with experimentally CET= $0.98 \mathrm{~nm}, \mathrm{SS}_{\text {for }}=42 \mathrm{mV} / \mathrm{dec}, \mathrm{SS}_{\text {rev }}=28 \mathrm{mV} / \mathrm{dec}$, switch-off $<0.2 \mathrm{~V}$, and hysteresis free strategies. In IEEE International Electron Devices Meeting (IEDM) 22-5 (IEEE, 2015).

32. Khan, A. I. et al. Negative capacitance in a ferroelectric capacitor. Nat. Mater. 14 182-186 (2015).

33. Dubourdieu, C. et al. Switching of ferroelectric polarization in epitaxial $\mathrm{BaTiO}_{3}$ films on silicon without a conducting bottom electrode. Nat. Nanotechnol. 8, 748-754 (2013).

34. Saeidi, A. et al. Negative capacitance as performance booster for tunnel FETs and MOSFETs: an experimental study. IEEE Electron Device Lett. 38, 1485-1488 (2017).
35. Zhang, Q., Zhao, W. \& Seabaugh, A. Low-subthreshold-swing tunnel transistors IEEE Electron Device Lett. 27, 297-300 (2006).

36. McGuire, F. A. et al. Sustained sub- $60 \mathrm{mV} /$ decade switching via the negative capacitance effect in $\mathrm{MoS}_{2}$ transistors. Nano Lett. 17, 4801-4806 (2017).

37. Si, M. et al. Steep-slope hysteresis-free negative capacitance $\mathrm{MoS}_{2}$ transistors Nat. Nanotechnol. 13, 24-28 (2018).

38. $\mathrm{Si}, \mathrm{M}$. et al. Steep-slope $\mathrm{WSe}_{2}$ negative capacitance field-effect transistor. Nano Lett. 18, 3682-3687 (2018).

39. Salahuddin, S. \& Datta, S. Can the subthreshold swing in a classical FET be lowered below $60 \mathrm{mV} /$ decade? In IEEE International Electron Devices Meeting (IEDM) 1-4 (IEEE, 2008).

40. Rusu, A., Saeidi, A. \& lonescu, A. M. Condition for the negative capacitance effect in metal-ferroelectric-insulator-semiconductor devices. Nanotechnology $\mathbf{2 7}$ 115201 (2016).

41. Khan, A. I., Yeung, C. W., Hu, C. \& Salahuddin, S. Ferroelectric negative capacitance MOSFET: capacitance tuning \& antiferroelectric operation. In International Electron Devices Meeting (IEDM) 11-3 (IEEE, 2011).

42. Saeidi, A., Jazaeri, F., Stolichnov, I. \& Ionescu, A. M. Double-gate negative-capacitance MOSFET with PZT gate-stack on ultra thin body SOI: an experimentally calibrated simulation study of device performance. IEEE Trans. Electron Devices 63, 4678-4684 (2016).

43. Khan, A. I., Radhakrishna, U., Chatterjee, K., Salahuddin, S. \& Antoniadis, D. A Negative capacitance behavior in a leaky ferroelectric. IEEE Trans. Electron Devices 63, 4416-4422 (2016).

44. Hoffmann, M. et al. Unveiling the double-well energy landscape in a ferroelectric layer. Nature 565, 464-467 (2019)

45. Sze, S. M. \& Ng, K. K. Physics of Semiconductor Devices (Wiley, 2006).

46. Salvatore, G. A. et al. Ferroelectric transistors with improved characteristics at high temperature. Appl. Phys. Lett. 97, 053503 (2010).

47. Tagantsev, A., Sherman, V., Astafiev, K., Venkatesh, J. \& Setter, N. Ferroelectric materials for microwave tunable applications. J. Electroceram. 11, 5-66 (2003).

48. Ginzburg, V. L. Phase transitions in ferroelectrics: some historical remarks. PhysicsUspekhi 44, 1037 (2001).

49. Zhou, D. et al. Electric field and temperature scaling of polarization reversal in silicon doped hafnium oxide ferroelectric thin films. Acta Mater. 99, 240-246 (2015).

50. Vopsaroiu, M., Blackburn, J., Cain, M. G. \& Weaver, P. M. Thermally activated switching kinetics in second-order phase transition ferroelectrics. Phys. Rev. B 82 024109 (2010).

51. Damjanovic, D. Ferroelectric, dielectric and piezoelectric properties of ferroelectric thin films and ceramics. Rep. Prog. Phys. 61, 1267 (1998).

52. Hoffmann, M., Ravindran, P. V. \& Khan, A. I. Why do ferroelectrics exhibit negative capacitance? Materials 12, 3743 (2019).

53. Castellanos-Gomez, A. et al. Deterministic transfer of two-dimensional materials by all-dry viscoelastic stamping. 2D Mater. 1, 011002 (2014).

54. Huang, Q. et al. Self-depleted T-gate Schottky barrier tunneling FET with low average subthreshold slope and high $\mathrm{I}_{\text {ON }} / \mathrm{l}_{\text {OFF }}$ by gate configuration and barrier modulation. In International Electron Devices Meeting (IEDM) 16-2 (IEEE, 2011).

55. Dey, A. W. et al. High-current GaSb/lnAs (Sb) nanowire tunnel field-effect transistors. IEEE Electron Device lett. 34, 211-213 (2013).

56. Bellando, F. et al. Subthermionic negative capacitance ion sensitive field-effect transistor. Appl. Phys. Lett. 116, 173503 (2020).

57. Saeidi, A. et al. Effect of hysteretic and non-hysteretic negative capacitance on tunnel FETs DC performance. Nanotechnology 29, 095202 (2018).

58. Jo, J. \& Shin, C. Negative capacitance field effect transistor with hysteresisfree sub-60-mV/decade switching. IEEE Electron Device Lett. 37, 245-248 (2016).

59. Enz, C., Chalkiadaki, M.-A. \& Mangla, A. Low-power analog/RF circuit design based on the inversion coefficient. In ESSCIRC Conference 2015-41st European Solid-State Circuits Conference (ESSCIRC) 202-208 (IEEE, 2015).

\section{ACKNOWLEDGEMENTS}

This publication has received funding from the European Research Council (ERC) under the European Union's Horizon 2020 research and innovation programme, grant agreement no. 695459, Milli-Tech.

\section{AUTHOR CONTRIBUTIONS}

S.K. and A.M.I. conceived the main idea of this experimental study on negative capacitance 2D/2D TFET, designed, and developed the process flow. S.K. performed all the fabrication processes, the electrical measurements and data analysis. C.G., A.S. 
and M.C. performed AFM and PFM measurements. S.K., C.G. and A.S. carried out the MFM characterization and optimization. S.K. and T.R. prepared the low temperature setup. S.K., A.S. and L.C. prepared the figures and schematics. S.K., A.S. and A.M.I. wrote the manuscript.

\section{COMPETING INTERESTS}

The authors declare no competing interests.

\section{ADDITIONAL INFORMATION}

Supplementary information The online version contains supplementary material available at https://doi.org/10.1038/s41699-021-00257-6.

Correspondence and requests for materials should be addressed to S.K.

Reprints and permission information is available at http://www.nature.com/ reprints
Publisher's note Springer Nature remains neutral with regard to jurisdictional claims in published maps and institutional affiliations.

(i) Open Access This article is licensed under a Creative Commons cc) Attribution 4.0 International License, which permits use, sharing, adaptation, distribution and reproduction in any medium or format, as long as you give appropriate credit to the original author(s) and the source, provide a link to the Creative Commons license, and indicate if changes were made. The images or other third party material in this article are included in the article's Creative Commons license, unless indicated otherwise in a credit line to the material. If material is not included in the article's Creative Commons license and your intended use is not permitted by statutory regulation or exceeds the permitted use, you will need to obtain permission directly from the copyright holder. To view a copy of this license, visit http://creativecommons. org/licenses/by/4.0/.

(c) The Author(s) 2021 